\title{
Düşük kütle oranlı iki değen çift yıldızın yörünge dönemi değişimlerinin incelenmesi: V404 Peg ve V619 Peg
}

\author{
Burcu ÖZKARDEŞ1,2,* \\ ${ }^{1}$ Çanakkale Onsekiz Mart Üniversitesi, Fen-Edebiyat Fakültesi, Uzay Bil. ve Tekn. Böl., Terzioğlu \\ Kampüsü, Çanakkale \\ ${ }^{2}$ Çanakkale Onsekiz Mart Üniversitesi, Astrofizik Araştırma Merkezi ve Ulupınar Gözlemevi, Çanakkale \\ Gelis Tarihi (Received Date): 26.06.2019 \\ Kabul Tarihi (Accepted Date): 09.09.2019
}

$\ddot{\mathbf{O} z}$

Bu çalışmada düşük kütle oranına sahip iki aşırı değen çift yıldızın yörünge dönemi değişimleri, detaylı olarak ilk kez incelenmiştir. Hedef sistemlerin dönem analizleri, veri tabanlarından ve literatür yayınlarından alınan minimum ışık zamanları kullanılarak $O$-C yöntemine göre yapılmıştır. V404 Peg'in O-C diyagramının aşağı parabol şeklinde

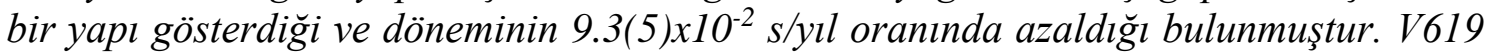

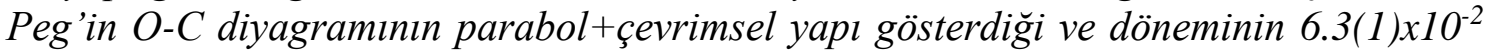
s/yıl oranında arttı̆̆ bulunmuş; çevrimsel dönem değişimi, olası üçüncü cisme ve Applegate mekanizmasına göre tartışılmıştır.

Anahtar kelimeler: Değen çift yıldızlar, dönem analizi, V404 Peg, V619 Peg.

\section{Investigation of orbital period variations of two low mass ratio contact binary stars: V404 Peg and V619 Peg}

\begin{abstract}
In this study the period variations of two over contact systems with low mass ratio are examined in detail for the first time. Period analyses of the systems are performed using the $O-C$ method with their all minima times collected from the databases and published papers. The O-C diagram of V404 Peg shows a downward parablic trend, and it is found that its period has a secular decrease at a rate of 9.3(5) $\times 10^{-2} \mathrm{~s} / \mathrm{yr}$. The $\mathrm{O}-\mathrm{C}$ diagram of V619 Peg shows an upward parabolic+cyclical character which indicates a continuous increase at a rate of $6.3(1) \times 10^{-2} \mathrm{~s} / \mathrm{yr}$ in the system's orbital period. The
\end{abstract}

\footnotetext{
*Burcu ÖZKARDEŞ, burcu@comu.edu.tr, http://orcid.org/0000-0002-6764-9299
} 
cyclical period variation is discussed according to a possible third companion and Applegate mechanism.

Keywords: Contact binary stars, period analysis, V404 Peg, V619 Peg.

\section{Giriş}

Örten çift yıldızların kimi sınıfları, yapılarının ve evrimlerinin incelenmesi bakımından araştırmacılara, görece ilgi çekici gelmektedir. Bu sınıflardan biri, W UMa türü örten çift sistemlerdir. W UMa türü çift yıldızların ışı eğrileri, eğrisel maksimumlara ve yaklaşık eşit derinlikli minimumlara sahiptir. Son söylenen durum, bu tür sistemlerde bileşen yıldızların yüzey sıcaklıklarının yaklaşık eşit olduğunu belirtir. Yörünge dönemleri kısa olup 0.2 gün $<P_{\text {yörünge }}<0.8$ gün aralığındadır. Bu sistemlerin tayf türleri, geç A'dan orta K’ya değişim göstermektedir. Geç tayf türünden sistemler olmaları nedeniyle konvektif bir atmosfere sahiptirler; dolayısıyla, yapılarında manyetik aktivite, leke gibi kromosferik ve koronal özellikleri barındırırlar [1]. Roche modeline göre W UMa türü çift yıldızlar, değen sistemler alt sınıfına girerler. Bu modele göre, her iki bileşen yıldız kendi Roche şişimlerini doldurmuştur. Öyle ki, bileşenlerin $\mathrm{L}_{1}$ Lagrange noktasından birbirlerine madde aktarması sonucu, bileşenleri saran ve uzanımı $\mathrm{L}_{2}$ Lagrange noktasına kadar gelebilen bir ortak zarf oluşur. Bu durumda, sistem aşırı değen çift yıldız olarak adlandırılır. Son yıllarda yapılan çalışmalar dikkate alındığında, bu tür sistemlerin kütle oranını (q) ve değme derecesi (f) parametresini temel alan bir sinıflama mevcuttur. Buna göre, W UMa türü çift yıldızlarda $\mathrm{q}<0.25$ ve $\mathrm{f} \geq \% 50$ olan sistemler, "düşük kütle oranlı derin değen çiftler" grubunu oluşturmaktadır [2]. Böylesi sistemler, W UMa türü çiftlerin bileşen yıldız çekirdeklerinin birleşimiyle oluştuğu düşünülen ve hızlı dönen tekil yıldızlar olan Mavi Aykırılar (Blue Stragglers) ile FK Com türü sistemlerin kökenini anlamada en önemli kaynaklardır. Bu çalışmada, şimdiye kadar, sadece doğrusal 1şık öğeleri belirlenmiş düşük kütle oranlı iki aşırı değen sistem olan V404 Peg'in ve V619 Peg'in yörünge dönemi değişimlerinin incelenmesi amaçlanmıştır. Hedef sistemlere ilişkin literatüre dayanan öz bilgiler, aşağıda verilmektedir.

"Semi-Automatic Variability Search" isimli proje kapsamında değişen olarak keşfedilen V404 Peg, elde edilen 1şık eğrisinin biçimine göre W UMa türü olarak sınıflandırılmıştır [3]. Aynı proje kapsamında, değişen olduğu belirlenen ve hedef sistem V404 Peg'i de içeren 9 sistemin 1şık eğrileri sunulmuştur [4]. V404 Peg ile birlikte iki değen çift yıldızın dikine hızları ölçülerek tayfsal yörünge çözümleri yapılmıştır; buradan, V404 Peg'in kütle oranı, 0.236 \pm 0.061 olarak elde edilmiştir [5]. Değişen Yıldızların 78. İsim Listesini içeren çalışmada, V404 Peg'in değişim türü EW olarak verilmiştir [6]. Sistemin BVRI 1şık ve dikine hız eğrileri analiz edilerek V404 Peg'in, $\mathrm{q}=0.243$ ve $\mathrm{f}=\% 32$ olmak üzere, A-türü aşırı değen çift yıldız olduğu ortaya konmuştur [7]. Bu çalışmada incelenen iki sistemin de dahil olduğu 100 değen çiftin parametreleri kullanılarak başlangıç kütlelerinin hesaplanması için yeni bir yöntem sunulmuştur [8]. W UMa türü çift yıldızların element bolluklarının belirlenmesini amaçlayan çalışmada, V404 Peg'in tayfsal verisinin az/duyarsız olması nedeniyle örnek sistem grubuna dahil edilmemiştir [9]. Hedef sistemin bazı fotometrik özellikleri, örten çift yıldızlara ilişkin katalog çalışmasında [10] yer alırken farklı yazarlarca kaydedilmiş minimum 1 şık zamanları da mevcuttur [11-14]. 
Literatürde az çalışılmış sistemlerden biri olan örten çift V619 Peg, ilk kez, NSVS (The Northern Sky Variability Survey) verileri kullanılarak 0.5553 gün döneme sahip bir W UMa türü çift yıldız olarak keşfedilmiştir [15]. 12 örten çift yıldızın minimum 1 şı zamanlarının verildiği çalışmada, V619 Peg için bir Min I ve bir Min II zamanı elde edilmiştir [16]. NSVS kataloğunda yer alan 4659 değişen sistemin sinıflaması yapılmış ve V619 Peg'in sınıfı, W UMa türü olarak belirlenmiştir [17]. Çift yıldız V619 Peg'in çok renk 1 şı ve dikine hız eğrileri analiz edilerek bileşen yıldızların fiziksel ve geometrik parametreleri verilmiş ve buna göre, sistem, düşük kütle oranlı A-türü W UMa çift yıldızı olarak tanımlanmıştır [18]. Örten çiftler V619 Peg'in ve V404 Peg'in verilerini kapsayan ve toplam yüz sistemin temel parametrelerini kullanan hesaplamaları içeren çalışma, W UMa türü değen sistemlerin yaşlarının ve yörünge evrimlerinin belirlenmesini amaçlamaktadır [19]. Ayrıca, 1022 parlak değen çift yıldızın fotometrik parametrelerini içeren katalog çalışmasında, yakın çift V619 Peg'in bazı temel parametreleri bulunmaktadır [20]. Tablo 1'de, incelenen sistemlere ilişkin temel bilgiler verilmektedir. Bu tabloda yer alan "mag." kisaltması, kadir biriminde parlaklığı temsil ederken “ $\odot$ ” sembolü, Güneş birimini karşılar.

Tablo 1. V404 Peg ve V619 Peg için temel bilgiler.

\begin{tabular}{|c|c|c|c|}
\hline Parametre & Birimi & V404 Peg & V619 Peg \\
\hline$V_{\mathrm{mag}}$ & mag. & 10.44 & 10.46 \\
\hline Tayf Türü & -- & F7V+F8V & F6V+F7V \\
\hline$M_{1} ; M_{2}$ & $\mathrm{M}_{\odot}$ & $1.18 ; 0.29$ & $1.38 ; 0.26$ \\
\hline$R_{1} ; R_{2}$ & $\mathrm{R}_{\odot}$ & $1.35 ; 0.71$ & $1.45 ; 0.71$ \\
\hline$a$ & $\mathrm{R} \odot$ & 2.67 & 2.64 \\
\hline Kaynak & -- & {$[7,21]$} & {$[18,21]$} \\
\hline
\end{tabular}

\section{Materyal ve Metot}

\subsection{Minimum ışık zamanları}

Hedef iki sistemin dönem değişimlerinin incelenmesi için, sadece, kullanıma açık olan veri tabanlarından (“ $O-C$ Gateway: http://var2.astro.cz/ocgate/index.php?lang=en” ve "BAV-Lichtenkecker: https://www.bav-astro.eu/index.php/veroeffentlichungen/servicefor-scientists/lkdb-engl") alınan minimum 1ş1k zamanlarıyla [7, 18] kaynaklarında verilen tutulma zamanlarının ortalama değerleri kullanılmıştır. Buna göre, her iki sistemin, CCD yöntemiyle elde edilen, sırasıyla, V404 Peg için 18 birinci minimum (Min I) + 16 ikinci minimum (Min II) olmak üzere toplam 34 tane, V619 Peg için 8 Min I +6 Min II olmak üzere toplam 14 tane tutulma zamanı bulunmaktadır. Sistemlerin minimum zaman bilgileri, sırasıyla, V404 Peg için Tablo 2'de, V619 Peg için Tablo 3 'te verilmektedir. Bu tablolarda kullanılan BAV-L ve OC kısaltmaları, sirasiyla, BAVLichtenkecker ve $O-C$ Gateway veri tabanlarını temsil ederken HJD ve CCD kısaltmaları ise, sırasıyla, Güneş merkezine indirgenmiş Jülyen gününü ve ChargeCoupled Device ifadesini karşılamaktadır. Ayrıca, [] işareti içinde verilen referansın ayrıntılı bilgisi, kaynaklar kısmında yer almaktadır. 
Tablo 2. V404 Peg'in minimum zaman bilgileri.

\begin{tabular}{|c|c|c|c|c|c|c|c|}
\hline $\begin{array}{c}\text { HJD } \\
(2400000+)\end{array}$ & $\begin{array}{l}\text { Min. } \\
\text { Türü }\end{array}$ & Yöntem & Referans & $\begin{array}{c}\text { HJD } \\
(2400000+)\end{array}$ & $\begin{array}{l}\text { Min. } \\
\text { Türü }\end{array}$ & Yöntem & Referans \\
\hline 52555.4988 & I & CCD & OC & 56120.8570 & II & CCD & BAV-L \\
\hline 55386.4555 & II & CCD & BAV-L & 56141.3945 & II & CCD & BAV-L \\
\hline 55411.8144 & I & CCD & BAV-L & 56905.3447 & I & CCD & BAV-L \\
\hline 55412.8636 & II & CCD & BAV-L & 56918.3404 & I & CCD & BAV-L \\
\hline 55500.2611 & I & CCD & {$[7]$} & 56918.5486 & II & CCD & BAV-L \\
\hline 55502.3576 & I & CCD & {$[7]$} & 56930.2874 & II & CCD & BAV-L \\
\hline 55503.4062 & II & CCD & {$[7]$} & 56930.4939 & I & CCD & BAV-L \\
\hline 55506.3400 & II & CCD & {$[7]$} & 57225.3901 & II & CCD & BAV-L \\
\hline 55511.3708 & II & CCD & {$[7]$} & 57241.5239 & I & CCD & BAV-L \\
\hline 55515.3522 & I & CCD & {$[7]$} & 57242.3637 & I & CCD & BAV-L \\
\hline 55424.8096 & I & CCD & BAV-L & 57242.5686 & II & CCD & BAV-L \\
\hline 55529.3916 & II & CCD & BAV-L & 57284.6954 & I & CCD & BAV-L \\
\hline 55793.4807 & II & CCD & BAV-L & 57336.6771 & I & CCD & BAV-L \\
\hline 55820.5145 & I & CCD & BAV-L & 57354.2746 & I & CCD & BAV-L \\
\hline 55851.3295 & II & CCD & BAV-L & 57590.4791 & II & CCD & BAV-L \\
\hline 55851.5345 & I & CCD & BAV-L & 57952.4399 & I & CCD & BAV-L \\
\hline 56109.5368 & II & CCD & BAV-L & 57981.3623 & I & CCD & OC \\
\hline
\end{tabular}

Tablo 3. V619 Peg'in minimum zaman bilgileri.

\begin{tabular}{|c|c|c|c|}
\hline $\begin{array}{c}\text { HJD } \\
(2400000+)\end{array}$ & $\begin{array}{c}\text { Min. } \\
\text { Türü }\end{array}$ & Yöntem & Referans \\
\hline 51549.6980 & I & CCD & OC \\
\hline 54045.4107 & II & CCD & OC \\
\hline 54046.3821 & I & CCD & OC \\
\hline 55049.4547 & II & CCD & {$[18]$} \\
\hline 55063.4481 & II & CCD & {$[18]$} \\
\hline 55066.3632 & I & CCD & {$[18]$} \\
\hline 55066.5579 & II & CCD & {$[18]$} \\
\hline 55153.6294 & II & CCD & OC \\
\hline 55501.7209 & I & CCD & OC \\
\hline 56239.6954 & II & CCD & OC \\
\hline 56929.0857 & I & CCD & OC \\
\hline 57316.2498 & I & CCD & OC \\
\hline 57624.8949 & I & CCD & OC \\
\hline 58365.0181 & I & CCD & OC \\
\hline
\end{tabular}

\subsection{O-C yöntemi}

Bir çift/çoklu sistemin dönemindeki değişimin varlığ 1 , iyi bilinen $O-C$ yöntemiyle ortaya konabilir. Burada, $O$ : Gözlenen minimum zaman1, $C$ : hesaplanan minimum zamanı ifade eder. $\mathrm{Bu}$ yöntemde, öncelikle, artıkların çevrim sayısına göre dağılımını gösteren $O-C$ diyagramı oluşturulur. Böylesi bir diyagramdaki dağınım, doğrusal, parabolik, çevrimsel ya da bu değişimlerin bir bileşimi şeklinde olabilmektedir. $O-C$ değerleri, aşağıda verilen Denklem 1 ile hesaplanmaktadır [22]. 


$$
\text { Min. } I=\mathrm{T}_{0}+\mathrm{PE}+\mathrm{QE}^{2}+\left(\frac{\mathrm{a}_{12} \operatorname{sini}}{\mathrm{c}}\left\{\frac{1-\mathrm{e}^{2}}{1+\mathrm{ecos} v} \sin (v+\omega)+\mathrm{ecos} \omega\right\}\right)
$$

Burada, $\mathrm{T}_{0}$ ve $\mathrm{P}$, başlangıç 1şık elemanlarını, E ve Q ise, sırasıyla, çevrim sayısını ve kuadratik terimi ifade eder. [23] tarafından verilen parantez içindeki bağıntı, sisteme çekimsel olarak bağlı üçüncü bileşenden kaynaklanan zamandaki gecikmeyi (LTTE: Light Time Travel Effect) tanımlamakta olup kullanılan terimlerin tanımları şöyledir: 1şık hızı c ile gösterilirken $a_{12} \operatorname{sini}$, i, e, $v$ ve w, çift yıldızın üçlü sistemin ortak kütle merkezi çevresinde çizdiği yörünge için sırasıyla, yarı-büyük eksen uzunluğun izdüşümünü, yörüngenin eğikliğini, yörüngenin basıklığını, yörüngenin gerçel anomali değerini ve yörüngede enberinin boylam açısını ifade eder. LTTE bağıntısında, $\mathrm{T}^{\prime}$ ve $\mathrm{P}_{12}$ gizli terimler olup üçlü sistemin yörüngesi için sırasıyla, enberiden geçiş zamanını ve dönemini belirtir.

\section{Bulgular}

İncelenen sistemlerin dönem analizleri, aşağıdaki iki alt başlıkta verilmiştir.

\subsection{V404 Peg}

Sistemin dönem analizi için Tablo 2'de verilen tüm Min I ve Min II zamanları kullanılmış ve bu zamanların $O-C$ değerleri, aşağıda verilen doğrusal 1 şık elemanları kullanılarak hesaplanmıştır [24].

Min. $I=2452555.5059+0 . .^{g} 41918108 x E$

Buradan, $O-C$ değerlerinin çevrim sayısına göre dağılımı, Şekil 1'de (üst panelde) gösterilmektedir. Diyagrama göre, sistemin döneminde düzenli bir azalışı temsil eden aşağı parabolik benzeri bir değişim gözlenmektedir. Analizde, parabolik yapıyı en iyi şekilde temsil etmek için Denklem 1'de parantez içindeki bağıntıyla verilen 1şık-zaman etkisi hesaba katılmamış ve tüm $O-C$ verilerine, en küçük kareler yöntemini kullanan ve [25] tarafından geliştirilen yazılım kodu uygulanmıştır. Minimum zamanların ağırlıkları, CCD gözlemi olması nedeniyle, 10 olarak girilmiştir. Elde edilen kuadratik 1şık elemanları, aşağıda verilmektedir. Parantez içindeki değerler, son basamağa göre standart hata değerleridir.

Min. $I=2452555.4981(377)+0 .^{g} 4191879(41) x E-6.17(35) x 10^{-10} x E^{2}$

Gözlem noktalarının kuramsal fit ile uyumu ve bu fitten kalan artıklar, çevrim sayısına göre Şekil 1'de gösterilmektedir. 


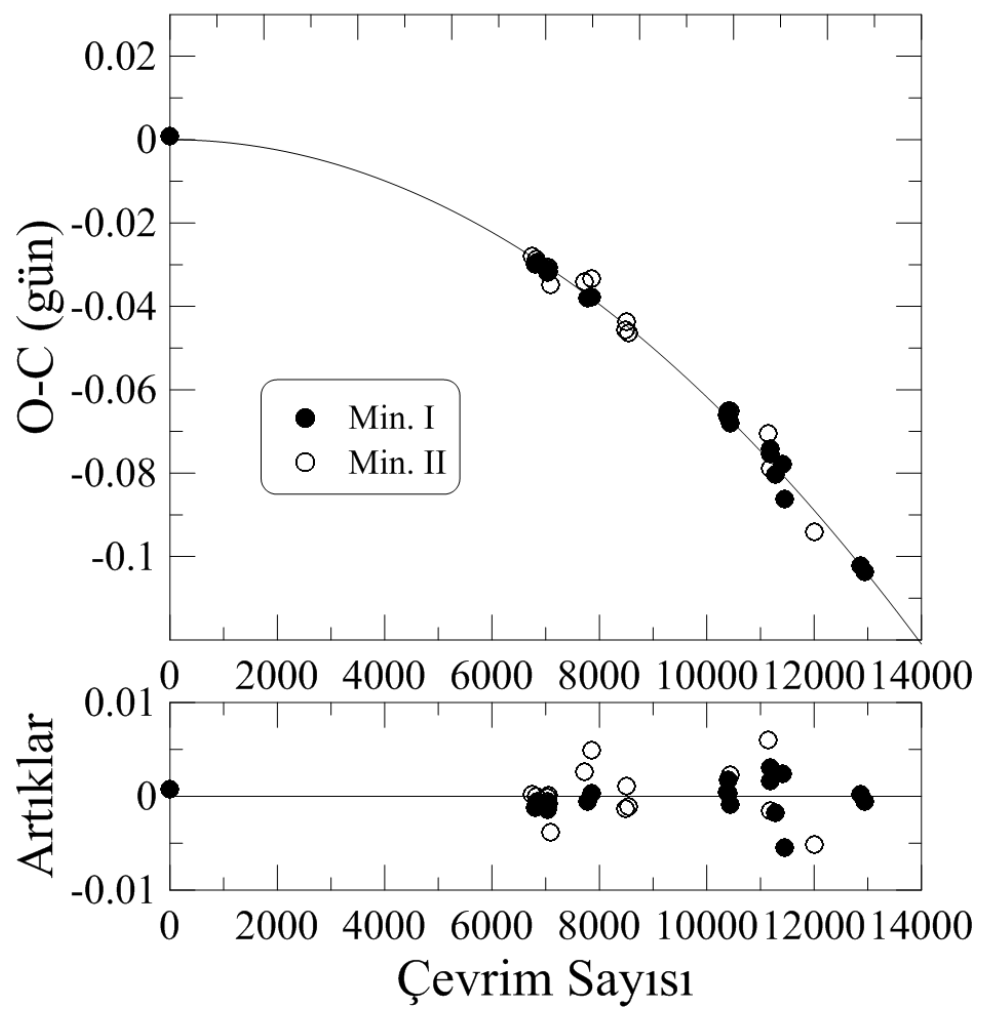

Şekil 1. Üst panel: Değen çift V404 Peg'in $O$ - $C$ verisinin dağılımı ve kuramsal eğriyle (düz çizgi) uyumu; Alt panel: Kuramsal eğriden kalan artıklar.

\subsection{V619 Peg}

Analizde, sistemin Tablo 3'te yer alan tüm minimum 1şık zamanları (Min I ve Min II) kullanılarak $O-C$ değerleri hesaplanmış; bunun için girdi parametreleri olarak [24]'ten alınan ve aşağıda Denklem 4 ile verilen doğrusal ışık elemanları kullanılmıştır.

Min. $I=2454299.8190+0 .{ }^{g} 38871515 x E$

Buna göre oluşturulan sistemin $O-C$ diyagramı, Şekil 2'de gösterilmekte olup genel dağılım, değen çift V619 Peg'in yörünge döneminde sürekli bir artışı destekleyen yukarı parabol biçimindedir. Böylesi bir değişimi temsil etmek amacıyla, öncelikle 1şıkzaman etkisi göz önüne alınmamış ve sistemin tüm $O-C$ verileri, Bölüm 3.1 'de bahsedilen yazılım kodu kullanılarak analiz edilmiştir. V404 Peg ile benzer şekilde, sistemin minimum zamanlarının ağırlıklarına 10 verilmiştir. Sonuç olarak, elde edilen en iyi kuramsal fiti karşılayan kuadratik ışık elemanları, aşağıdaki gibidir.

Min. $I=2454299.8206(42)+0 .^{g} 3887124(8) x E+3.77(20) x 10^{-10} x E^{2}$

Standart hata değerleri, parantez içinde verilmiş olup son basamağa göre düzenlenmiştir. Sadece parabolik yaklaşım altında yapılan analizde, elde edilen $\chi^{2}$ değeri 0.0005 'dir. Şekil 2'de, gözlemsel verinin bu kuramsal modelle karşılaştırılması ve artıkları gösterilmektedir. 
Y1l

19992002200520082011201420172020

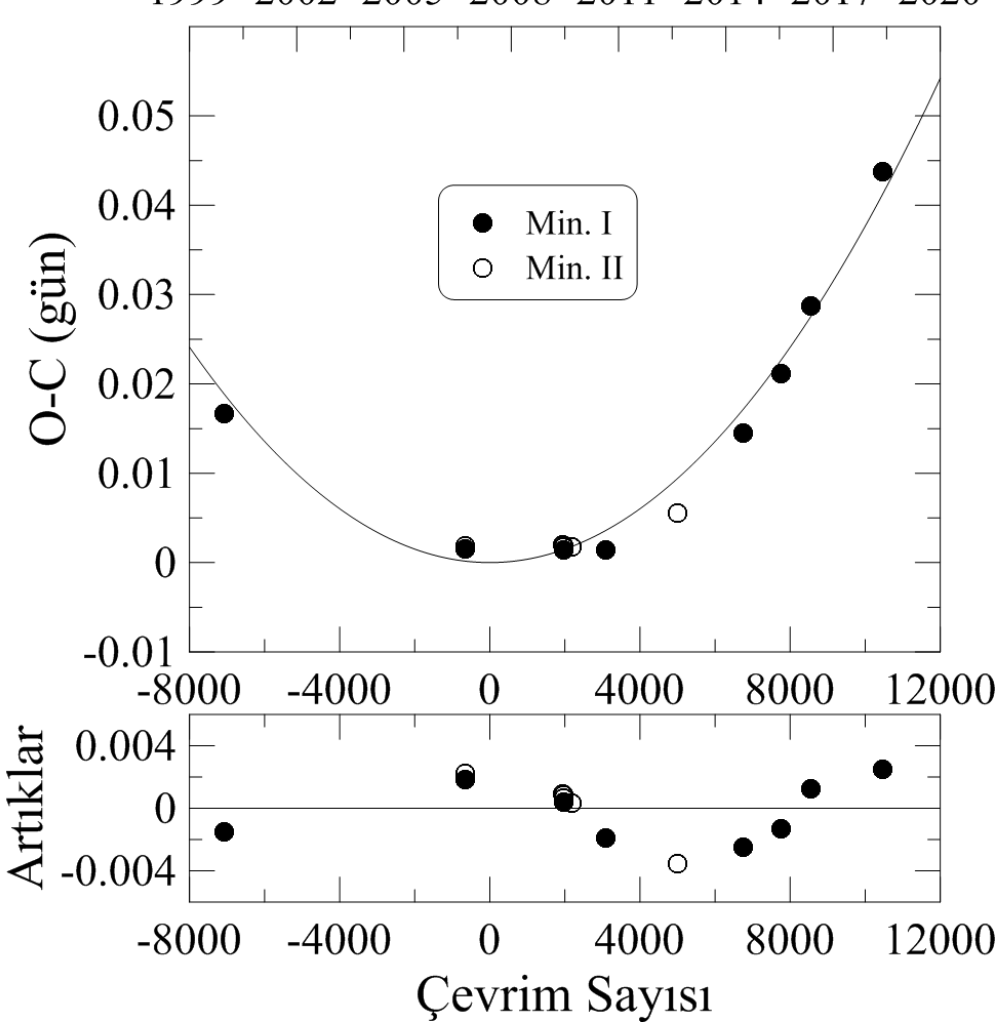

Şekil 2. Değen çift V619 Peg'in $O-C$ değişiminin kuramsal modelle (düz çizgi) karşılaştırılması (üst panel) ve artıklar (alt panel).

Şekil 2'nin alt panelinde, artıkların dağılımının sinüzoidal bir yapıda olduğu görülmektedir. Böylesi bir değişimin nedeni, çoğunlukla, sistemde görülmeyen üçüncü cisim kaynaklı olduğu kabul edilmektedir. Bu kabul altında, sistemin $O-C$ verilerine, ışık-zaman etkisini de içeren Denklem 1, yine aynı yazılım kodu aracılığıyla uygulanmıştır. Buradan, $\chi^{2}=0.0001$ olarak elde edilmiştir. İki $\chi^{2}$ değeri karşılaştırıldığında, parabolik+çevrimsel yaklaşımın gözlemsel verilerle daha uyumlu olduğu görülmektedir. Sonuç olarak V619 Peg'in $O-C$ diyagramı, parabol üzerine binmiş sinüzoidal/çevrimsel yapıyla temsili ve en iyi uyum eğrisinden kalan artıklar, Şekil 3'te gösterilirken sistemin çevrimsel $O-C$ değişimi ve kuramsal modelle temsili, Şekil 4'te gösterilmektedir. 
Y11

19992002200520082011201420172020

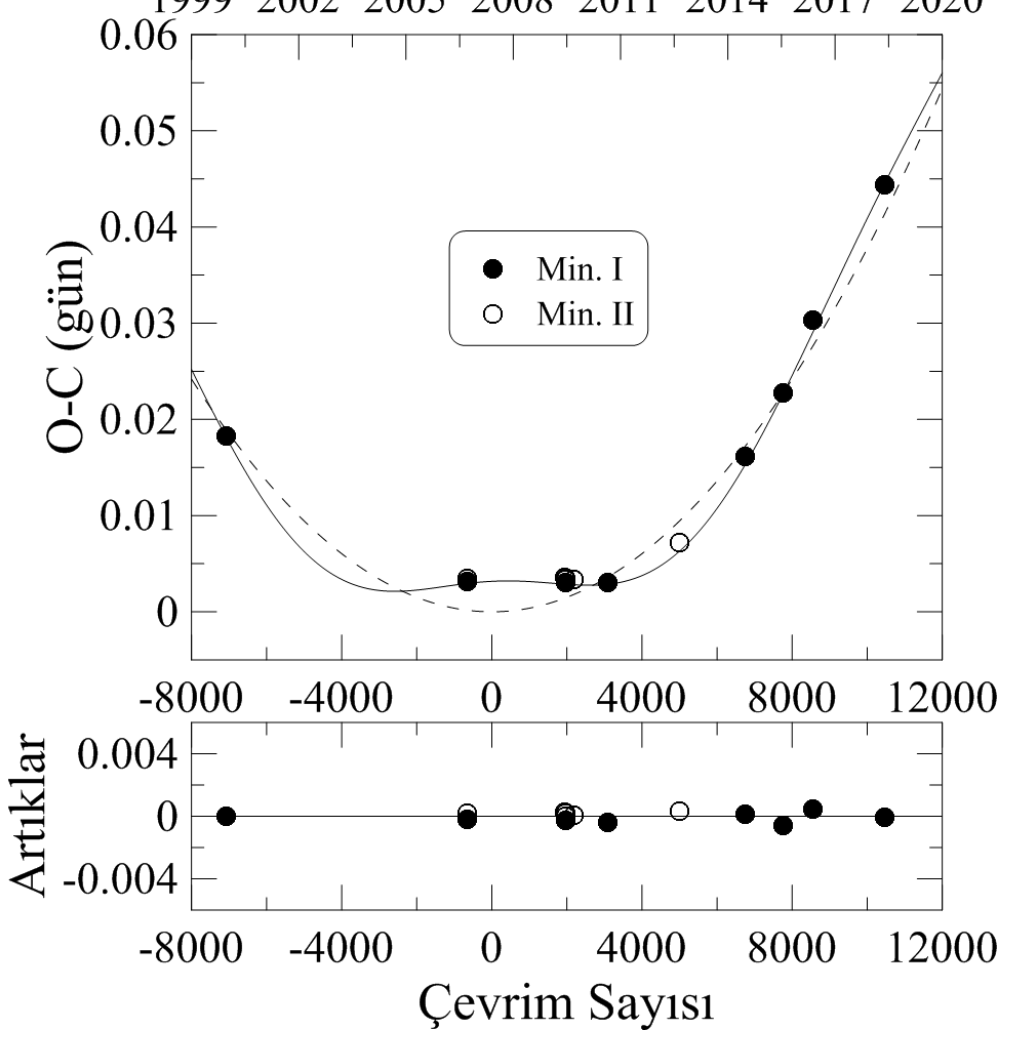

Şekil 3. Değen çift V619 Peg'in $O-C$ verisinin parabol (kesikli çizgi) üzerine binmiş çevrimsel yapı (düz çizgi) ile temsili (üst panel) ve en iyi fitten kalan artıklar (alt panel).

Y1l

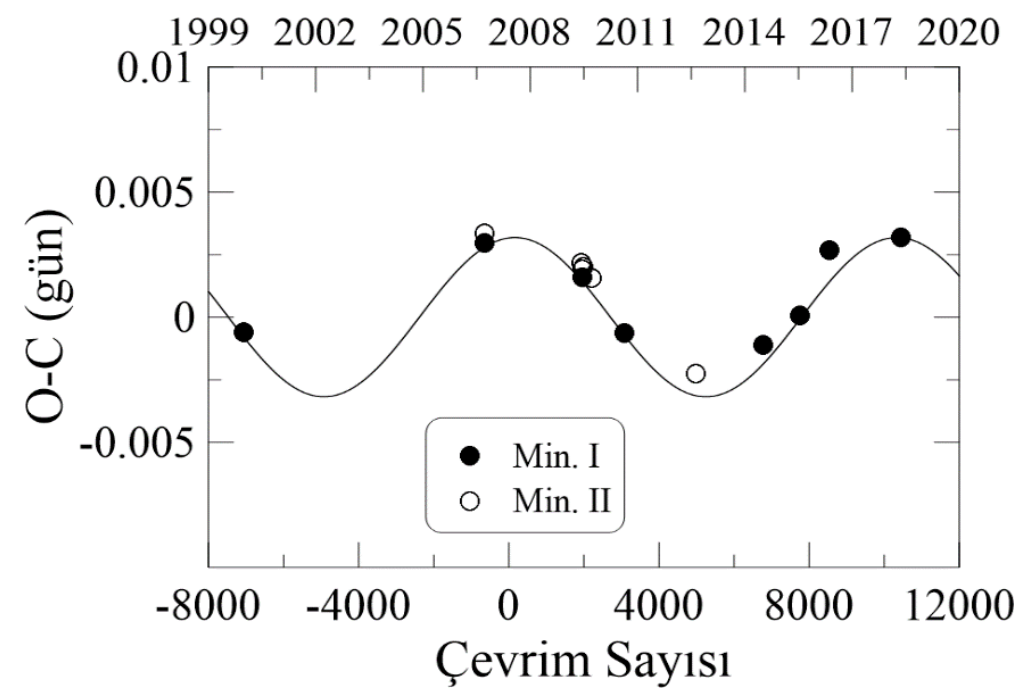

Şekil 4. V619 Peg'in çevrimsel $O-C$ değişimi ve kuramsal modelle temsili (düz çizgi). 


\section{Sonuçlar ve tartışma}

$\mathrm{Bu}$ çalışmada, Pegasi takımyıldızında yer alan düşük kütle oranlı iki aşırı değen sistemin, V404 Peg ve V619 Peg, yörünge dönemi değişimi, detaylı olarak ilk kez incelenmiştir. Elde edilen sonuçlar, şu şekildedir:

V404 Peg'in $O-C$ analizine göre yörünge dönemi azalmakta olup dönemdeki azalma oranı $(\mathrm{d} P / \mathrm{dt})$, Denklem 3'te verilen quadratik/parabol terimi kullanılarak, 9.3(5) $\times 10^{-2}$ s/yıl olarak hesaplanmıştır. Böylesi bir azalışın nedeni, büyük kütleli bileşenden küçük kütleli bileşene aktarılan maddeden kaynaklanmaktadır [26]. Sistemin toplam kütlesinin ve açısal momentumunun korunduğu varsayımı altında, aktarılan maddenin miktarının $\left(\dot{m}_{1}\right)$ hesaplanmasında Denklem 6'da verilen bağıntı kullanılmıştır [27]. V404 Peg'in dönem analizinden elde edilen sonuçlar, son basamağa göre düzenlenmiş standart hata değerleriyle birlikte, Tablo 4'te verilmektedir. Tabloda, $\mathrm{M}_{\odot}$ ve AB birimi, sirasiyla, Güneş kütlesini ve astronomi birimini temsil ederken HJD ise, Güneş merkezli Jülyen gününü ifade eder.

$\frac{\Delta P}{P}=\frac{3 \dot{\mathrm{m}}_{1}\left(\mathrm{~m}_{1}-\mathrm{m}_{2}\right)}{\mathrm{m}_{1} \mathrm{~m}_{2}}=3 \frac{1-\mathrm{q}^{2}}{\mathrm{qM}} \dot{\mathrm{m}}_{1}$

Tablo 4. V404 Peg'in dönem analizinden elde edilen parametreleri ve değerleri.

\begin{tabular}{|c|c|c|}
\hline Parametre & Birimi & Değeri \\
\hline$T_{0}$ & $\begin{array}{c}\text { HJD } \\
(+2400000)\end{array}$ & $52555.4981(377)$ \\
\hline$P_{\text {yörünge }}$ & Gün & $0.4191879(41)$ \\
\hline$Q$ & Gün & $-6.17(35) \times 10^{-10}$ \\
\hline $\mathrm{d} P / \mathrm{dt}$ & $\mathrm{s} / \mathrm{y} 1 \mathrm{l}$ & $9.3(5) \times 10^{-2}$ \\
\hline$\dot{m}_{1}$ & $\mathrm{M}_{\odot} / \mathrm{y} 1 \mathrm{l}$ & $3.29 \times 10^{-7}$ \\
\hline
\end{tabular}

Literatürde, değen sistemlere ilişkin dönem analizi çalışmalarında, örneğin [28, 29], kütle aktarım oranı $10^{-7}-10^{-8} \mathrm{M}_{\odot} / \mathrm{y}$ ll değer aralığındadır. Buradan, hesaplanan kütle aktarım oranı değerinin literatürle uyumlu olduğu söylenebilir.

V619 Peg'in dönem değişimi, iki yaklaşım altında incelenmiştir: Parabol ve Parabol+Çevrimsel. $\chi^{2}$ testine göre, ikinci yaklaşımın, gözlemleri daha iyi temsil ettiği sonucuna varılmıştır. Buna göre, örten değen çift V619 Peg'in dönem analiziyle belirlenen parametreleri ve değerleri, Tablo 5'te verilmektedir. Burada, standart hata, birim ve kısaltma bilgisi, Tablo 4'tekiyle aynıdır.

Tablo 5. V619Peg'in dönem analizinden elde edilen parametreleri ve değerleri.

\begin{tabular}{|c|c|c|c|c|c|}
\hline Parametre & Birimi & Değeri & Parametre & Birimi & Değeri \\
\hline$T_{0}$ & $\begin{array}{c}\text { HJD } \\
(+2400000)\end{array}$ & $54299.8198(6)$ & $e$ & -- & 0.0 \\
\hline$P_{\text {yörünge }}$ & Gün & $0.38871236(11)$ & $w$ & Derece & 90 \\
\hline$Q$ & Gün & $3.78(1) \times 10^{-10}$ & $T$ & $\begin{array}{c}\text { HJD } \\
(+2400000)\end{array}$ & $5463(344)$ \\
\hline $\mathrm{d} P / \mathrm{dt}$ & $\mathrm{s} / \mathrm{y} 1 \mathrm{l}$ & $6.3(1) \times 10^{-2}$ & $P_{12}$ & $\mathrm{Y}_{1} 1$ & $10.8(2)$ \\
\hline $\mathrm{d} M / \mathrm{dt}$ & $\mathrm{M} \odot / \mathrm{y} 1 \mathrm{l}$ & $1.93 \times 10^{-7}$ & $f\left(\mathrm{~m}_{3}\right)$ & $\mathrm{M}_{\odot}$ & $0.0014(5)$ \\
\hline $\mathrm{a}_{12} \operatorname{sini}$ & $\mathrm{AB}$ & $0.55(9)$ & $\begin{array}{c}\mathrm{m}_{3} \\
\left(i=90^{\circ} \mathrm{için}\right)\end{array}$ & $\mathrm{M}_{\odot}$ & $0.17(7)$ \\
\hline
\end{tabular}


Çift yıldızlarda, bileşenlerden biri ya da her ikisi geri tayf türüne (F5 ve daha geri tayf türüne) sahipse, $O-C$ diyagramında gözlenen çevrimsel değişimin olası nedeni, manyetik aktivite kaynaklı olabilir ki böylesi bir değişim, Applegate mekanizmasıyla açıklanır [30]. V619 Peg, geri tayf türüne sahip bir sistemdir (bknz Tablo 1). Toplam 1şıtmaya katkısı daha fazla olan baş bileşenin manyetik aktiviteden sorumlu olduğu kabulüyle, V619 Peg için hesaplanan Applegate modeli parametreleri ve değerleri, Tablo 6'da verilmiştir. Bu modele göre, manyetik aktivite nedeniyle ortaya çıkan çevrimsel dönem değişimi için, aktiviteden sorumlu bileşenin yüzey altı manyetik alan değeri $\mathrm{B} \sim 10 \mathrm{kG}$ olmas1 gerekir. Elde edilen sonuçlar $\left(\mathrm{B}_{\mathrm{V} 619 \mathrm{Peg}}=12.6 \mathrm{kG}\right)$, model için verilen değer aralığında olup uyumludur.

Tablo 6. V619 Peg'in Applegate modeline göre elde edilen parametreleri ve değerleri.

\begin{tabular}{|c|c|c|c|c|c|c|}
\hline Sistem & $\begin{array}{c}\Delta \mathrm{J} \\
(\mathrm{erg} / \mathrm{s})\end{array}$ & $\Delta \Omega / \Omega$ & $\begin{array}{c}\Delta \mathrm{E} \\
(\mathrm{erg})\end{array}$ & $\begin{array}{c}\mathrm{I}_{\mathrm{s}} \\
\left(\mathrm{gcm}^{2}\right)\end{array}$ & $\begin{array}{c}\Delta \mathrm{L}_{\mathrm{rms}} \\
\left(\mathrm{L}_{\odot}\right)\end{array}$ & $\begin{array}{c}\mathrm{B} \\
(\mathrm{kG})\end{array}$ \\
\hline V619 Peg & $2.96 \times 10^{47}$ & $1.18 \times 10^{-3}$ & $3.26 \times 10^{40}$ & $1.34 \times 10^{54}$ & 0.076 & 12.60 \\
\hline
\end{tabular}

Her iki sistem için yapılan dönem analizlerinin sonucu, ortaya konan bileşenler arası madde aktarımının, olası üçüncü cismin varlığının ve manyetik aktivite çevriminin araştırılması için daha fazla minimum ışı zamanlarının elde edilmesine, bunun yanısıra; farklı dalga boylarında (manyetik aktivite için özellikle mor öte, X-1şın gibi) yapılmış duyarlı fotometrik, tayfsal ve astrometrik gözlemlere önemle ihtiyaç vardır.

\section{Teşekkür}

Çalışmada, "NASA Astrophysics Data System Abstract Service" ve "SIMBAD at CDS, Starsbourg, France" olmak üzere iki veri kaynağından yararlanılmıştır. Düzenlenmiş yazılım kodunun kullanılmasındaki desteğinden dolayı Dr. P. Zasche'ye ve bu çalışmayı değerlendirerek önerilerde bulunan değerli hakemlere teşekkür ederim.

\section{Kaynaklar}

[1] Bilir, S., Karataş, Y., Demircan, O. ve Eker, Z., Kinematics of W Ursae Majoris Type Binaries and Evidence of Two Types of Formation, Monthly Notices of the royal Astronomical Society, 357(2), 497-517, (2005).

[2] Qian, S., Yang, Y., Zhu, L., Liying, H. ve Jiajia, Y. J., Photometric studies of twelve deep, low-mass ratio overvontact binary systems, Astrophysics and Space Science, 304, 25-28, (2006).

[3] Maciejewski, G., Karska, A. ve Niedzielski, A., GSC 02757-00769 - A new EW binary system, Information Bulletin on Variable Stars, No. 5370, 1, (2003).

[4] Maciejewski, G. ve Niedzielski, A., A semi-automatic variability search, Baltic Astronomy, 13, 700-703, (2004).

[5] Maciejewski, G. ve Ligeza, P., Mass ratio determination of binary systems BD+14d 5016, GSC 2757-769 and GSC 3472-641, Information Bulletin on Variable Stars, No. 5504, 1, (2004).

[6] Kazarovets, E. V., Samus, N. N., Durlevich, O. V., Kireeva, N. N. ve Pastukhova, E. N., The $78^{\text {th }}$ name-list of variable stars, Information Bulletin on Variable Stars, No. 5721, 1, (2006). 
[7] Gürol, B., Terzioğlu, Z., Gürsoytrak, S. H., Gökay, G. ve Derman, E., Absolute and geometric parameters of the W UMa-type contact binary V404 Pegasi, Astronomische Nachricten, 332, 690-696, (2011).

[8] Y1ld1z, M. ve Doğan, T., On the origin of W UMa type contact binaries-a New method for computation of initial masses, Monthly Notices of the Royal Astronomical Society, 430, 2029-2038, (2013).

[9] Rucinski, S. M., Pribulla, T. ve Budaj, J., Spectroscopic metallicity determinations for W UMa type binary stars, The Astronomical Journal, 146,1-20, (2013).

[10] Avvakumova, E. A., Malkov, O. Y. ve Kniazev, A. Y., Eclipsing variables: catalogue and classification, Astronomische Nachricten, 334, 860-865, (2013).

[11] Huebscher, J. ve Monninger, G., BAV-results of observations-photoelectric minima of selected eclipsing binaries and maxima of pulsating stars, Information Bulletin on Variable Stars, No. 5959, 1, (2011).

[12] Huebscher, J., Lehmann, P. B. ve Walter, F., BAV-results of observationsphotoelectric minima of selected eclipsing binaries and maxima of pulsating stars, Information Bulletin on Variable Stars, No. 6010, 1, (2012).

[13] Huebscher, J., BAV-results of observations-photoelectric minima of selected eclipsing binaries and maxima of pulsating stars, Information Bulletin on Variable Stars, No. 6152, 1, (2015).

[14] Huebscher, J., BAV-results of observations-photoelectric minima of selected eclipsing binaries, Information Bulletin on Variable Stars, No. 6157, 1, (2016).

[15] Nicholson, M., Sutherland, J. ve Sutherland, C., New variable stars found in the NSVS database, Open European Journal on Variable Stars, 12, 1-3, (2005).

[16] Gürol, B., Derman, E., Müyesseroğlu, Z., Gürdemir, L., Gökay, G., Özbek, N., Sağır, U., Kalcı, R., Salman, G., Çoker, G., Eminoğlu, B., Demircan, Y. ve Terzioğlu, Z., Minima times of some eclipsing binary stars, Information Bulletin on Variable Stars, No. 5791, 1, (2007).

[17] Hoffman, D. I., Harrison, T. E. ve McNamara, B. J., Automated variable stars classification using the Northern Sky Variability Survey, The Astronomical Journal, 138, 466-477, (2009).

[18] Gürol, B., Derman, E., Saguner, T., Gürsoytrak, S. H., Terzioğlu, Z., Gökay, G., Demircan, Y., Okan, A. ve Saral, G., Absolute and geometric parameters of W UMa type contact binary TYC 1174-344-1, New Astronomy, 16, 242-249, (2011).

[19] Y1ldz, M., Origin of W UMa-type contact binaries-age and orbital evolution, Montly Notices of the Royal Astronomical Society, 437, 185-194, (2014).

[20] Gettel, S. J., Geske, M. T. ve McKay, T. A., A catalog of 1022 bright contact binary stars, The Astronomical Journal, 31, 621-632, (2006).

[21] SIMBAD Astronomical Database, http://simbad.u-strasbg.fr/simbad, (22.05.2019).

[22] Yakut, K., İbanoğlu, C., Kalemoni, B. ve Değirmenci, Ö. L., New light curve analysis and period changes of the overcontact binary $\mathrm{XY}$ Leonis, Astronomy\&Astrophysics, 401, 1095-1100, (2003).

[23] Irwin, J. B., Standard light-time curves, The Astronomical Journal, 64, 149155, (1959).

[24] Kreiner, J. M., Up-to-date linear elements of eclipsing binaries, Acta Astronomica, 54, 207-210, (2004). 
[25] Zasche, P., Liakos, A., Niarchos, P., Wolf, M., Manimanis, V. ve Gazeas, K., Period changes in six contact binaries: WZ And, V803 Aql, DF Hya, PY Lyr, FZ Ori, and AH Tau, New Astronomy, 14, 121-128, (2009).

[26] Huang, S. S., A dynamical problem in binary systems and its bearing on stellar evolution, The Astronomical Journal, 61, 49-61, (1956).

[27] Kwee, K. K., Investigation of variations in the period of sixteen bright shortperiod eclipsing binary stars, Bulletin of the Astronomical Institutes of the Netherlands, 14, 131-151, (1958).

[28] Pribulla, T., Chochol, D., Rovithis-Livaniou, H. ve Rovithis, P., The contact binary AW Ursae Majoris as a member of a multiple system, Astronomy and Astrophysics, 345, 137-148, (1999).

[29] Kriwattanawong, W., Tasuya, O. ve Poojon, P., Period change investigation of the lowmass ratio binary BO Ari, New Astronomy, 44, 12-16, (2016).

[30] Applegate, J. H., A mechanism for orbital period modulation in close binaries, The Astrophysical Journal, 385, 621-629, (1992). 